Corrigendum

\title{
Corrigendum to "Kuntai Capsule plus Hormone Therapy vs. Hormone Therapy Alone in Patients with Premature Ovarian Failure: A Systematic Review and Meta-Analysis"
}

\author{
Weiping Liu $\left(\mathbb{D},{ }^{1}\right.$ Truong-Nam Nguyen, ${ }^{2}$ Thu-Van Tran Thi, ${ }^{2}$ and Shaohu Zhou $\mathbb{D}^{3}$ \\ ${ }^{1}$ The First Clinical College of Guangzhou University of Chinese Medicine, Guangzhou 510405, China \\ ${ }^{2}$ Vietnam University of Traditional Medicine, Hanoi 100000, Vietnam \\ ${ }^{3}$ The First Affiliated Hospital, Guangzhou University of Chinese Medicine, Guangzhou 510120, China \\ Correspondence should be addressed to Shaohu Zhou; zhoushaohu@vip.163.com
}

Received 7 December 2020; Accepted 7 December 2020; Published 2 February 2021

Copyright ( 92021 Weiping Liu et al. This is an open access article distributed under the Creative Commons Attribution License, which permits unrestricted use, distribution, and reproduction in any medium, provided the original work is properly cited.

In the article titled "Kuntai Capsule plus Hormone Therapy vs. Hormone Therapy Alone in Patients with Premature Ovarian Failure: A Systematic Review and Meta-Analysis" [1], errors have been identified in the forest plots (Figures 4 and 5).
The correct position of the $X$-axis labels, "Kuntai Capsule + Hormone" and "Hormone," should be the opposite of their current position. The corrected Figures 4 and 5 are as follows.

\begin{tabular}{|c|c|c|c|c|c|c|c|c|c|c|}
\hline \multirow{3}{*}{$\begin{array}{l}\text { Study or subgroup } \\
\text { H.F,Yuan et al. } 2018\end{array}$} & \multicolumn{2}{|c|}{ Kuntai Capsule + Hormone } & \multicolumn{2}{|c|}{ Hormone } & \multirow{2}{*}{$\begin{array}{l}\text { Weight } \\
(\%)\end{array}$} & \multirow{2}{*}{$\begin{array}{c}\text { Odds ratio } \\
\text { M-H, fixed, } 95 \% \text { CI }\end{array}$} & \multirow{2}{*}{\multicolumn{4}{|c|}{$\begin{array}{c}\text { Odds ratio } \\
\text { M-H, fixed, } 95 \% \text { CI }\end{array}$}} \\
\hline & Events & Total & Events & Total & & & & & & \\
\hline & 36 & 41 & 28 & 40 & 9.7 & $3.09[0.97,9.79]$ & & & & \\
\hline H.P.Li et al. 2018 & 37 & 39 & 28 & 39 & 4.0 & $7.27[1.49,35.45]$ & & & & \\
\hline J Guo 2017 & 53 & 54 & 46 & 54 & 2.4 & $9.22[1.11,76.49]$ & & & & \\
\hline Q.H.Deng et al. 2017 & 48 & 50 & 39 & 50 & 4.4 & $6.77[1.42,32.37]$ & & & & \\
\hline R.Z.Li 2014 & 46 & 55 & 35 & 55 & 16.1 & $2.92[1.19,7.19]$ & & & $\Longrightarrow$ & \\
\hline S.Q.Kang 2018 & 36 & 39 & 29 & 39 & 6.3 & $4.14[1.04,16.44]$ & & & & \\
\hline S.R.Pan et.al 2015 & 47 & 53 & 41 & 52 & 13.2 & $2.10[0.71,6.18]$ & & & 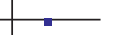 & \\
\hline S.Y.Zeng et al. 2014 & 36 & 39 & 29 & 39 & 6.3 & $4.14[1.04,16.44]$ & & & & \\
\hline X.H.Zhou 2018 & 37 & 41 & 28 & 41 & 7.7 & $4.29[1.26,14.60]$ & & &. & \\
\hline X.L.Gao 2017 & 38 & 41 & 34 & 41 & 7.0 & $2.61[0.62,10.89]$ & & & & \\
\hline X.Q.Yang 2017 & 59 & 63 & 51 & 63 & 9.1 & $3.47[1.05,11.43]$ & & & & \\
\hline Y.Y.Wu et al. 2016 & 68 & 75 & 53 & 75 & 13.9 & $4.03[1.60,10.15]$ & & & $\longrightarrow$ & \\
\hline Total $(95 \%$ CI) & & 590 & & 588 & 100.0 & $3.76[2.65,5.35]$ & & & $>$ & \\
\hline Total events & 541 & & 441 & & & & & & & \\
\hline \multicolumn{7}{|c|}{ Heterogeneity: $\mathrm{chi}^{2}=3.80, d f=11(P=0.98) ; I^{2}=0 \%$} & 0.005 & 0.1 & 10 & 200 \\
\hline \multicolumn{7}{|c|}{ Test for overall effect: $Z=7.41(P<0.00001)$} & & Hormone & Kuntai Capsu & ule + Hormone \\
\hline
\end{tabular}

FIGURE 4: Meta-analysis results for the total effective treatment rate. 


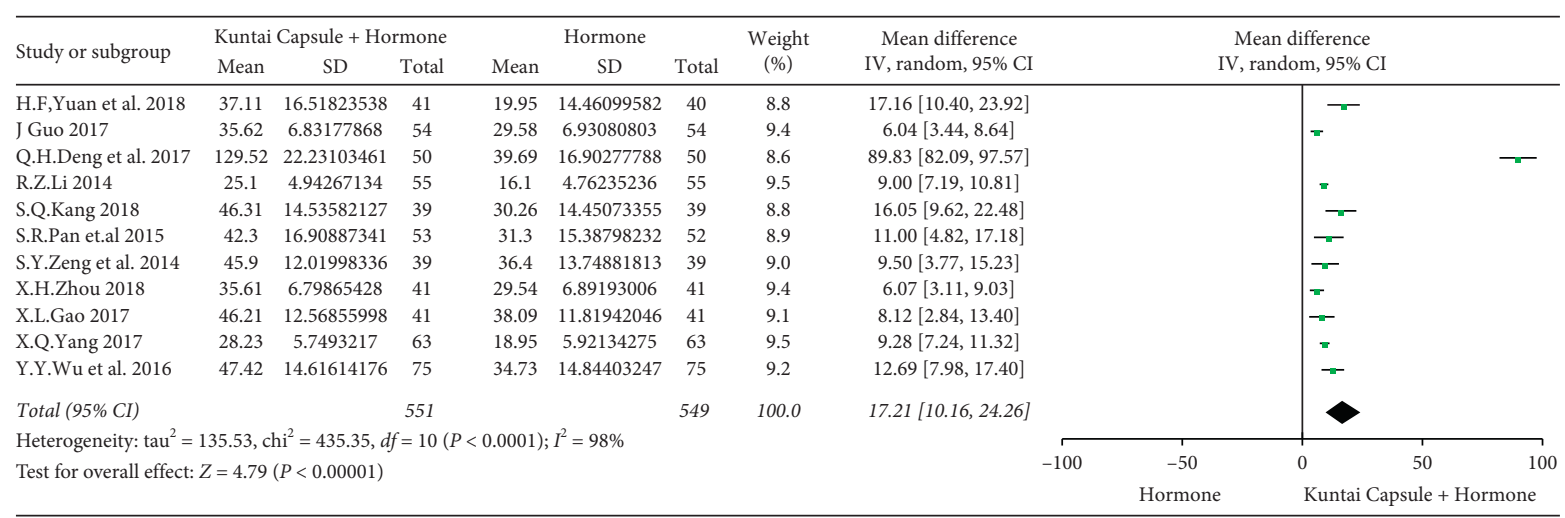

FIgure 5: Meta-analysis results for the E2 levels.

\section{Acknowledgments}

The authors would like to acknowledge Dr. Jingyan Song and Dr. Zhengao Sun from the Shandong University of Traditional Chinese Medicine, China, for identifying this concern.

\section{References}

[1] W. Liu, T.-N. Nguyen, T.-V. Tran Thi, and S. Zhou, "Kuntai capsule plus hormone therapy vs. hormone therapy alone in patients with premature ovarian failure: a systematic review and meta-analysis," Evidence-Based Complementary and Alternative Medicine, vol. 2019, Article ID 2085804, 9 pages, 2019. 